\title{
Estudo Correlacional entre Interesses Profissionais e Autoeficácia com Tecnólogos
}

\author{
Demerval Rogério Masotti* \\ Faculdade de Tecnologia de Jundiaí - Centro Paula Souza, Jundiaí, SP, Brasil \\ Ana Paula Porto Noronha \\ Universidade São Francisco, Itatiba, SP, Brasil
}

\begin{abstract}
RESUMO
No contexto da orientação profissional, interesses profissionais e autoeficácia são construtos de grande importância para o autoconhecimento dos orientandos. O presente estudo teve por objetivo verificar as relações entre os construtos junto a tecnólogos. Participaram 172 estudantes, com idades entre 19 e 57 anos. Os instrumentos utilizados foram a Escala de Aconselhamento Profissional (EAP) e a Escala de Autoeficácia para Atividades Ocupacionais (EAAOc). Os resultados indicaram correlações entre os interesses profissionais e a autoeficácia com magnitudes variando entre baixas e altas; para os interesses profissionais e as fontes de autoeficácia, muitos coeficientes variaram de baixos a muito baixos. A pesquisa possibilitou identificar outras evidências de validade, recomendando-se novos estudos com tecnólogos de diferentes cursos e instituições de ensino.
\end{abstract}

Palavras-chave: interesses profissionais; autoeficácia; orientação profissional; testes psicológicos.

\begin{abstract}
Correlational Study between Vocational Interests and Self-Efficacy with Technologists

In the context of vocational guidance, professional interests and self-efficacy constructs are of great importance to the self-knowledge of the advisees. The present study aimed to verify the relations between the constructs with technologists. 172 students participated, aged between 19 and 57 years. The instruments used were the "Escala de Aconselhamento Profissional" (EAP) and the "Escala de Autoeficácia para Atividades Ocupacionais" (EAAOc). The results indicated correlations between professional interests and self-efficacy with magnitudes ranging from low to high; to the professional interests and sources of self-efficacy, many coefficients ranged from low to very low. This research allowed us to identify other evidence of validity, but further studies with technologists from different courses and educational institutions are recommended.
\end{abstract}

Keywords: vocational interests; self-efficacy; vocational guidance; psychological tests.

Escolher uma profissão ou ocupação é um grande desafio inerente aos indivíduos em diferentes momentos da vida e, por esse motivo, o tema tem sido investigado por pesquisadores da área de orientação profissional (Melo-Silva, Leal, \& Fracalozzi, 2010; Noronha, Sisto, \& Santos, 2010; Teixeira, Lassance, Silva, \& Bardagi, 2007; entre outros). Ao longo dos anos o mercado de trabalho tem se revelado cada vez mais exigente e complexo, o que requer uma preparação para corresponder a tais expectativas. No que se refere ao jovem em momento de transição (escola-trabalho ou escola-ensino superior/técnico), e para que ela ocorra satisfatoriamente são necessários investimentos e decisões mais imediatas, devido à relevância e complexidade da tomada de decisão. É importante ressaltar que escolher adequadamente uma ocupação ou profissão implica benefícios às pessoas e, consequentemente, à sociedade (Noronha, Sisto, \& Santos, 2007).

No presente século, a orientação profissional, tanto no Brasil quanto no exterior, está passando por transformações, conforme pontuado por Sparta, Bardagi e Teixeira (2006). Isso se deve em virtude das demandas decorrentes do mercado de trabalho, que possui

* Endereço para correspondência: Demerval Rogério Masotti - prof.demerval@fatec.sp.gov.br 
novas características, como estruturas enxutas, e uso de recursos tecnológicos. Nesse contexto, a orientação profissional tem como finalidade não apenas informar a respeito das profissões, mas também complementar o trabalho de autoconhecimento e das variáveis do ambiente que envolve a escolha em si.

De acordo com Levenfus e Soares (2002), a orientação profissional no contexto brasileiro é comumente vista como auxiliar no processo de escolha de uma profissão ou carreira, uma vez que ela procura conciliar a necessidade pessoal com a demanda do mercado de trabalho. Diante disso, a informação é um dos principais componentes a ser considerado durante a escolha profissional, envolvendo a percepção que o indivíduo tem de si e do ambiente.

Noronha et al. (2007) afirmam que os psicólogos, quando possível e necessário, podem fazer uso de instrumentos de avaliação psicológica durante o processo de orientação profissional. Observa-se que por meio deles, torna-se factível conhecer o indivíduo, sendo assim possível fornecer elementos que auxiliem no processo de tomada de decisão. Mais especialmente, no que diz respeito à avaliação psicológica na área de orientação profissional, o uso de testes busca fornecer informações que deverão auxiliar na ampliação do autoconhecimento ou na tomada de decisão profissional (American Educational Research Association, American Psychological Association \& National Councilon Measurement in Education, AERA, APA $\&$ NCME, 1999). Isto posto, tem-se que o objeto de investigação desta pesquisa é avaliar a relação entre dois construtos psicológicos, quais sejam, interesses profissionais e autoeficácia, que foram analisados respectivamente por meio dos instrumentos Escala de Aconselhamento Profissional (EAP) e Escala de Autoeficácia para Atividades Ocupacionais (EAAOc).

Embora os instrumentos possuam evidências de validade, tal como preconizado pela AERA, APA e NCME (1999), novos estudos devem ser realizados, tendo em vista os objetivos/contextos das avaliações e as amostras. Assim, pretende-se buscar evidência de validade baseada na relação com outras variáveis, cujo objetivo é a análise da associação dos escores do teste com variáveis externas, tal como preconizado pela APA, AERA e NCME (1999). Esperam-se relações significativas, contudo de baixas magnitudes entre eles, com a amostra de tecnólogos, ainda não pesquisada com os respectivos instrumentos.
A justificativa para a realização da pesquisa se dá também com base nas Resoluções do Conselho Federal de Psicologia (CFP, 2001, 2003) as quais informam que, com o objetivo de possibilitar o atendimento ao fim proposto, os instrumentos devem atestar seus parâmetros psicométricos. Assim, tanto os novos instrumentos lançados no mercado profissional, quanto aqueles que já se encontram em uso, devem passar por verificações sistemáticas de seus parâmetros psicométricos, a fim de garantir a qualidade científica.

Em relação aos interesses profissionais, um dos construtos por ora estudados, Savickas (1995) afirma que eles podem ser interpretados como uma interface entre o indivíduo e o ambiente. É considerado um construto motivacional, já que se trata de um estado que confere energia ao sujeito e o direciona rumo a um objetivo. Dessa forma, tem-se que os interesses funcionam como ponte por meio da qual os objetivos são alcançados, as necessidades satisfeitas, em função das gratificações pretendidas.

Adicionalmente, o autor afirma que ao avaliar os interesses e os adotar como indicadores de personalidade possibilita obter informações sobre as necessidades, valores e preferências dos indivíduos. Ainda, destaca que interesse, no latim, significa "entre ser/estar". Portanto, o construto representa o elo entre o indivíduo e o mundo e, dessa forma, os interesses favoreceriam a adaptação às condições ambientais, em um processo de autocompletamento, à medida que ajudariam na manutenção da integridade do indivíduo. A partir disso, evidencia-se a relevância de se estudar esse construto em processos de orientação profissional, uma vez que a ampliação do autoconhecimento sobre o tema pode ajudar os orientandos no planejamento do seu futuro profissional.

Lent, Brown e Hackett (1994), ao elaborarem o modelo da Teoria Sócio-Cognitiva do Desenvolvimento de Carreira (TSCDC), afirmam que a formação de interesses profissionais é a primeira de três etapas intrinsecamente relacionadas, as quais possibilitam compreender o desenvolvimento profissional. A segunda etapa é uma consequência da anterior e trata das escolhas acadêmicas e profissionais, já que nessa fase ocorrerá a definição dos cursos a serem realizados, assim como, a área de atuação profissional. Enquanto isso, a terceira refere-se ao desempenho e persistência em atividades educacionais e profissionais. Em todo o processo, ocorre o monitoramento da per- 
formance do sujeito, com base nos resultados conquistados, e a realimentação do processo, ou seja, o indivíduo poderá reforçar ou redefinir suas preferências.

Ainda, com base no modelo TSCDC, os interesses profissionais são definidos como padrões de preferências, rejeições e indiferenças a profissões e atividades relacionadas à carreira. Lent et al. (1994) afirmam que as pessoas observam outras realizando diversas tarefas profissionais e, também, realizam experiências a partir de atividades lúdicas, acadêmicas e culturais, as quais poderão servir de reforço. Esse conjunto de ações viabiliza o desenvolvimento de percepções nas pessoas de que podem fazer determinadas atividades melhor do que outras e, em função disso, criam a expectativa de conquistar resultados a partir da execução de certas atividades, o que influenciará a formação dos interesses profissionais. Dessa maneira, os interesses e as habilidades podem se desenvolver ao longo da vida. Supõe-se que a escolha da profissão seja influenciada diretamente pelos fatores relacionados às vivências dos sujeitos, assim como por meio da interação com fatores externos, dentre eles, pressão social e aspectos econômicos, que consequentemente influenciam o nível e o conteúdo das escolhas realizadas.

Bueno, Lemos e Tomé (2004) e Levenfus e Soares (2002) afirmam que é possível estimar o quanto de satisfação um sujeito será capaz de experimentar no desempenho de uma profissão; isso se torna possível a partir de um conhecimento adequado dos interesses de um indivíduo. Os autores também comungam da concepção de que os interesses são tidos como estados motivacionais, caracterizados por despertar a atenção e direcionar o foco a certos objetos e atividades. Em razão disso, há a possibilidade de despertarem sentimentos de agrado ou desagrado provocando atitudes de atração ou repulsa diante dos objetos.

Em relação à autoeficácia, Betz e Borgen (2000) afirmam que esse construto possui a função de mediar o comportamento de escolha por meio de três indicadores: o primeiro deles trata dos comportamentos de aproximação versus evitação; o segundo refere-se à qualidade do desempenho no comportamento; e, por fim, o terceiro está relacionado à persistência diante de obstáculos ou experiências que neutralizam as crenças. No que diz respeito ao comportamento de aproximação, é necessário enfatizar que ele é especialmente importante no contexto de decisão de carreira, em razão de estar relacionado às atividades, cursos ou profissões que o indivíduo quer tentar ou irá perseguir. No entanto, as ações de evitação possivelmente surjam nas situações em que as crenças de autoeficácia são reduzidas, levando à eliminação de opções profissionais. Ainda a este respeito, Nunes e Noronha (2009) dissertam que há pouca probabilidade de um forte interesse surgir em áreas de atuação em que ocorra crença desfavorável de autoeficácia, ou em que ocorram expectativas de resultado negativas ou neutras.

Diante disso, a autoeficácia é um dos elementos constitutivos que assumem uma posição central no processo de escolha e no desenvolvimento de carreira. Entende-se que ela é algo que colabora na previsão dos interesses específicos dos alunos, no rendimento escolar nas disciplinas de sua preferência, nas várias possibilidades de atuação profissional, bem como na persistência e no sucesso obtido na área escolhida conforme as afirmações de Bandura (1977; 1986; 1997; 2001); Pajares (2002) e Lent et al. (1994).

Até este ponto foram apresentados os conceitos referentes aos construtos interesses e autoeficácia. $\mathrm{Na}$ sequência serão apresentados os resultados de pesquisas realizadas com a EAP, que é o principal foco deste estudo, uma vez que se busca para ela evidência de validade baseada na relação com outras variáveis.

Com o objetivo de verificar a magnitude das correlações existentes entre interesses profissionais de pais e filhos, e destes com as fontes de eficácia percebidas, Noronha e Ambiel (2008), contaram com 70 participantes, sendo 35 jovens e um dos genitores. A EAP foi aplicada em conjunto com a Escala de Autoeficácia para Atividades Ocupacionais (EAAOc). As correlações entre as dimensões da EAP, segundo as respostas de pais e filhos, demonstraram que Ciências Exatas, Artes e Comunicação, e Ciências Humanas e Sociais Aplicadas se correlacionaram de forma significativa entre si exibindo magnitudes variando entre baixas e moderadas. Além disso, os escores dos filhos em Ciências Biológicas e da Saúde se correlacionaram com os escores dos genitores em Atividades Burocráticas e Entretenimento apresentando magnitudes baixas. No que diz respeito às relações entre as fontes de eficácia e os interesses, a Experiência Direta da EAAOc se correlacionou significativamente com os interesses dos filhos em Atividades Burocráticas, enquanto Aprendizagem Vicária, com Ciências Agrárias e Ambientais, e Ciências Biológicas e da Saúde. To- 
davia, o único fator da EAAOc que apresentou coeficiente de magnitude moderada com os interesses dos pais foi Persuasão Verbal com a dimensão Ciências Exatas.

Nunes e Noronha (2011) com o objetivo de analisar a relação entre a autoeficácia e suas fontes e os interesses, usaram a EAAOc e o Self-Directed Search (SDS) em 289 participantes, e a EAAOc e a EAP em 107. Foram encontradas correlações significativas e baixas entre autoeficácia e interesses, como por exemplo entre Artístico (EAAOc) e Realista (SDS), Social (EAAOc) e Ciências Exatas (EAP) e, também, Social (EAAOc) e Ciências Biológicas e da Saúde (EAP). Também foram encontrados coeficientes entre as fontes de autoeficácia e os interesses, com magnitudes baixas, entre Experiência Direta (EAAOc) e tipo Artístico (SDS) e, também, Persuasão Verbal (EAAOc) e Artes e Comunicação (EAP).

Foi realizado um estudo por Sartori, Noronha, Godoy e Ambiel (2010) cujo objetivo foi avaliar as correlações entre as dimensões da EAP e as seções Competências, Carreiras e Habilidades do Self-Directed Search Career Explorer (SDS). Participaram da pesquisa 132 estudantes provenientes de 3 escolas particulares do interior de São Paulo. Os resultados demonstraram correlações estatisticamente significativas $(p<0,05)$ entre alguns tipos do SDS e dimensões da EAP, os quais são teoricamente relacionados como a que ocorreu entre Artes e Comunicação (EAP) e Artístico (SDS) $(r=0,54)$ na seção Competências (conjunto de competências exigidas ou possíveis de serem aprendidas para o exercício de dadas ocupações) do SDS. Já na seção Carreiras (conjunto de nomes de diversificadas atividades ocupacionais) também identificaram uma correlação alta $(r=0,62)$ para Artes e Comunicação (EAP) e Artístico (SDS). Por fim, na seção Habilidades recebeu destaque a correlação moderada $(r=0,48)$ entre Atividades Burocráticas (EAP) e Convencional (SDS).

Pode-se verificar a partir das pesquisas apresentadas que a EAP foi testada com amostras distintas. Assim, o presente estudo é inédito, uma vez que trata das relações entre interesses profissionais e a autoeficácia com tecnólogos, os quais (Brasil, 2010a; Brasil, 2010b) realizam cursos superiores de curta duração, possibilitando que conquistem um título de graduação rapidamente. Além disso, tem como característica principal a formação específica para atuarem na pro- dução e inovação científico-tecnológica, e para a gestão de processos de produção de bens e serviços, bem como dar continuidade aos estudos em nível de pósgraduação. Dessa forma, trata-se de um grupo com formação profissional diferente dos participantes de outras pesquisas realizadas. A seguir, a descrição do método.

\section{MÉTODO}

\section{Participantes}

Participaram 172 estudantes, sendo 102 do sexo masculino $(59,3 \%)$ e 70 do feminino $(40,7 \%)$, com idades variando entre 19 e 57 anos (Média=27,64 e $\mathrm{DP}=9,24)$. Em relação aos cursos, 80 alunos estavam matriculados em Tecnologia em Informática para a Gestão de Negócios (46,5\%), outros 76 cursavam Tecnologia em Logística e Transportes $(44,2 \%)$ e 16 em Tecnologia em Eventos (9,3\%). No que se refere aos semestres letivos, 105 participantes estavam no $5^{\circ}$ semestre $(61 \%)$ e os demais 67 encontravam-se no $6^{\circ}$ semestre (39\%). Ao analisar os períodos, foi possível observar que a maior concentração ocorreu no noturno com 107 estudantes $(62,2 \%) ; 35$ (20,3\%) pertenciam ao diurno e, por fim, no vespertino 30 alunos $(17,4 \%)$. Os participantes eram provenientes de uma instituição pública de nível superior do interior do estado de São Paulo.

\section{Instrumentos}

\section{EAP - Escala de Aconselhamento Profissional (No- ronha \& cols., 2007)}

Objetiva avaliar os interesses profissionais. O instrumento possui 61 itens, que são respondidos em uma escala tipo Likert de cinco pontos (de 1 a 5), em termos de quanto o estudante gostaria de realizar certas atividades.

Após a construção, a escala foi validada com uma amostra de 762 estudantes universitários de ambos os sexos, sendo 59\% mulheres, com faixa etária entre 17 e 73 anos, pertencentes a 13 cursos distintos de graduação. O instrumento é composto por sete dimensões, cujos índices de saturação dos itens foram superiores a 0,30 e explicaram 57,31\% da variância. As dimensões são Ciências Exatas (14 itens), Artes e Comunicação (14 itens), Ciências Biológicas e da Saúde (9 itens), Ciências Agrárias e Ambientais (13 itens), Atividades 
Burocráticas (13 itens), Ciências Humanas e Sociais Aplicadas (10 itens), e, por fim, Entretenimento (6 itens).

Como evidências de validade de critério, foram comparadas as médias obtidas pelos participantes em cada dimensão em relação ao curso de cada um, com o intuito de identificar quais carreiras poderiam ser diferenciadas em cada dimensão. Os estudos de evidência de validade de critério possibilitaram verificar que as preferências retratadas pelas várias dimensões permitiram distinguir os cursos estudados, ainda que carreiras diferentes possam se assemelhar quanto às suas preferências em algumas dimensões. A precisão do instrumento (Alfa de Cronbach) variou entre 0,79 e 0,94 e os de Spearman-Brown e Guttman entre 0,75 e 0,91 (Noronha et al., 2007).

\section{EAAOc - Escala de Autoeficácia para Atividades Ocupacionais (Nunes \& Noronha, 2009)}

O instrumento está organizado em duas seções, sendo que a primeira busca identificar a autoeficácia para atividades ocupacionais, avaliadas na escala tipo Likert de 5 pontos, variando entre pouca confiança (valores mais próximos de 1) e muita confiança (valores mais próximos de 5) na capacidade pessoal para realizar atualmente as atividades ocupacionais listadas. Essa parte da escala possui 126 atividades ocupacionais, sendo que foram usados 21 itens para cada tipo. A segunda parte da escala avalia as fontes de autoeficácia, sendo que as respostas podem variar entre 1 (menor concordância) e 5 (maior concordância), totalizando 58 itens, agrupados da seguinte forma: 16 itens para avaliar a experiência pessoal, 13 para aprendizagem vicária, 13 para a persuasão verbal e 16 para os indicadores fisiológicos.

A escala foi aplicada em 1.020 estudantes do ensino médio, sendo $56,7 \%$ alunos de escola pública; dos estados de São Paulo e Mato Grosso; com média de idade de 16,3 anos ( $\mathrm{DP}=2,31$ ); sendo $56,4 \%$, mulheres. A autoeficácia para atividades ocupacionais é avaliada por meio de seis fatores com base no modelo RIASEC proposto por Holland (1959) e Holland, Fritzsche e Powell (1994), quais sejam Realista, Investigativo, Artístico, Social, Empreendedor e Convencional; os quais explicaram $46,8 \%$ da variância com alfas de Cronbach superiores a 0,86. As fontes de autoeficácia foram organizadas em dois fatores, ou seja, experiências autênticas - esse fator concentra 3 fontes: experiência pessoal, persuasão verbal e indicadores fisiológicos - que explicaram $31,6 \%$ da variância (al$\mathrm{fa}=0,95)$ e a aprendizagem vicária, $8,4 \%$ (alfa=0,87).

\section{Procedimentos}

Foi feito contato com a instituição de ensino, requisitando autorização para a aplicação dos instrumentos de pesquisa. Após o recebimento da autorização por parte da instituição de ensino, o projeto foi submetido à análise de um Comitê de Ética de uma instituição de ensino superior. Tendo recebido parecer favorável, solicitou-se aos participantes a autorização para aplicação dos testes, mediante a assinatura do TCLE - Termo de Consentimento Livre e Esclarecido - e, em seguida, deu-se início à coleta de dados. A aplicação aconteceu de forma coletiva, nas próprias salas de aulas da instituição, tendo sido iniciada pela EAP. A duração foi de aproximadamente 40 minutos.

\section{RESULTADOS E DISCUSSÃO}

A seguir serão apresentadas as informações referentes às análises estatísticas, as quais foram realizadas para atender os objetivos da presente pesquisa: verificar a relação entre os Interesses Profissionais e a Autoeficácia para Atividades Ocupacionais, com vistas à busca de evidências de validade, baseada na relação entre outras variáveis. Também serão exibidas as respectivas discussões. A Tabela 1 apresenta os valores das correlações entre as dimensões dos interesses profissionais da EAP e os fatores de autoeficácia da primeira seção da EAAOc.

De 42 correlações possíveis, apenas 9 não apresentaram significância estatística. Foram identificados coeficientes variando de $r=0,19$ a $r=0,79$, portanto, de baixos a altos (Sisto, 2007). Tomando como referência a preferência por Ciências Exatas, observou-se associação significativa com quatro fatores da autoeficácia, quais sejam, Realista, Investigativa, Convencional e Empreendedora. No entanto, as duas mais fortes se deram com Realista e Investigativa, o que é possível explicar sob a perspectiva da construção de ambos os instrumentos. Mais especificamente, de acordo com Noronha et al. (2007), a dimensão de Ciências Exatas é composta por atividades como montar bancos de dados digitais, divulgar e vender softwares, analisar e interpretar dados numéricos, dentre outras. Com isso, supõe-se que a correlação significativa tenha se dado em virtude dos indivíduos associa- 
dos ao fator Realista (EAAOc) demonstrarem maior confiança na realização de atividades com características concretas, como as realizadas de forma prática no dia a dia, as quais apresentam uma tendência ao pouco uso de habilidades sociais ou sensibilidade diante dos problemas (Nunes, 2009; Holland, 1959; Holland et al., 1994).

Os resultados corroboram os encontrados por Sartori e cols. (2010), que obtiveram correlação moderada entre Ciências Exatas e o tipo Realista, indicando características comuns entre dimensão e fator, respectivamente. Ainda a este respeito, Holland (1977) afirma que o tipo Realista está associado com profissões da área de exatas, tais como engenharia, matemática, física, dentre outras, o que também explicaria a corre- lação moderada com a autoeficácia investigativa, que é a crença no exercício de atividades que lhe permitam expressar sua inclinação analítica e imaginativa, além da dedicação aos projetos científicos (Nunes \& Noronha, 2011). Duas outras correlações com magnitudes menores foram encontradas, com autoeficácia Empreendedora e Convencional. Possivelmente, algumas características comuns dos tipos e da dimensão (Ciências Exatas) podem ter explicado o coeficiente baixo, como por exemplo, a preferência do Convencional por atividades organizadas, de economia e aritmética, além da pequena habilidade nas relações interpessoais, o que também é característico dos que preferem as exatas.

Tabela 1

Correlação de Pearson (r) entre as Dimensões da EAP e a Autoeficácia da EAAOc ( $N=172)$

\begin{tabular}{|c|c|c|c|c|c|c|c|}
\hline \multirow{2}{*}{ Dimensões da EAP } & & \multicolumn{6}{|c|}{ Fatores da EAAOc } \\
\hline & & Realista & Investigativa & Artística & Social & Empreendedora & Convencional \\
\hline \multirow{2}{*}{ Ciências Exatas } & $r$ & 0,64 & 0,46 & 0,002 & 0,03 & 0,19 & 0,28 \\
\hline & $p$ & $0,000 * *$ & $0,000 * *$ & 0,980 & 0,718 & $0,012^{*}$ & $0,000 * *$ \\
\hline \multirow{2}{*}{ Artes e Comunicação } & $r$ & 0,09 & 0,11 & 0,79 & 0,35 & 0,23 & $-0,04$ \\
\hline & $p$ & 0,269 & 0,146 & $0,000 * *$ & $0,000 * *$ & $0,002^{*}$ & 0,591 \\
\hline \multirow{2}{*}{$\begin{array}{l}\text { Ciências Biológicas } \\
\text { e da Saúde }\end{array}$} & $r$ & 0,22 & 0,36 & 0,25 & 0,53 & 0,25 & 0,21 \\
\hline & $p$ & $0,005^{*}$ & $0,000^{* *}$ & $0,001^{*}$ & $0,000 * *$ & $0,001^{*}$ & $0,005^{*}$ \\
\hline \multirow{2}{*}{$\begin{array}{c}\text { Ciências Agrárias e } \\
\text { Ambientais }\end{array}$} & $r$ & 0,19 & 0,38 & 0,16 & 0,52 & 0,30 & 0,33 \\
\hline & $p$ & $0,015^{*}$ & $0,000 * *$ & $0,036^{*}$ & $0,000 * *$ & $0,000 * *$ & $0,000 * *$ \\
\hline \multirow{2}{*}{ Atividades Burocráticas } & $r$ & 0,33 & 0,26 & $-0,06$ & 0,33 & 0,51 & 0,69 \\
\hline & $p$ & $0,000 * *$ & $0,000 * *$ & 0,468 & $0,000 * *$ & $0,000 * *$ & $0,000 * *$ \\
\hline \multirow{2}{*}{$\begin{array}{l}\text { Ciências Humanas e } \\
\text { Sociais Aplicadas }\end{array}$} & $r$ & $-0,05$ & 0,25 & 0,43 & 0,59 & 0,35 & 0,29 \\
\hline & $p$ & 0,479 & $0,001^{*}$ & $0,000 * *$ & $0,000 * *$ & $0,000 * *$ & $0,000 * *$ \\
\hline \multirow{2}{*}{ Entretenimento } & $r$ & 0,01 & 0,05 & 0,51 & 0,50 & 0,39 & 0,21 \\
\hline & $p$ & 0,864 & 0,479 & $0,000 * *$ & $0,000 * *$ & $0,000 * *$ & $0,007^{*}$ \\
\hline
\end{tabular}

$* p<0,05 ; * * p<0,001$

No que se refere à dimensão Artes e Comunicação da EAP, foram identificados três coeficientes significativos, embora um tenha particular importância por ser o maior da análise realizada $(r=0,79)$, qual seja, o que expressa a relação com à autoeficácia artística. A dimensão Artes e Comunicação implica interesse por desenhar; dublar; produzir desfiles, catálogos, editoriais de moda e campanhas publicitárias; criar, mixar e editar trilhas sonoras; coordenar a apresentação de um espetáculo, por exemplo, tal como pontuado por Noronha et al. (2007). Ao lado disso, a maior crença no fator Artístico está compreendida pela segurança na realização de atividades que enfatizam o uso da sensi- 
bilidade e criatividade preterindo atividades concretas e burocráticas, buscando solucionar as questões diárias por meio da autoexpressão em atividades artísticas e evitando situações estruturadas de alto contato social ou grande esforço físico (Nunes, 2009; Holland, 1959; Holland et al., 1994). Assim como ocorreu com a dimensão anterior, na presente foram obtidos dois coeficientes menores, embora significativos, com os fatores Social e Empreendedor da EAAOc. A preferência por atividades artísticas como teatro, pintura, música, literatura e organização de festas, assim como o uso dos sentimentos, mais do que a razão, são alguns exemplos das possíveis aproximações entre Artes e Comunicação (EAP) e o Social (Holland, Fritzsche \& Powell, 1994). Quanto ao fator Empreendedor (EAAOc), não se encontrou hipóteses teoricamente sustentadas.

Ao analisar as relações entre a dimensão Ciências Biológicas e da Saúde e o fator Social (EAAOc), observou-se significância estatística com todos os fatores. No entanto, apenas com o fator Social a magnitude foi moderada. De acordo com Noronha e cols. (2007) a dimensão da EAP compreende atividades como realizar cirurgias, fazer pesquisas genéticas, investigar a natureza e a causa de doenças. Por sua vez, o fator Social (Nunes, 2009; Holland, 1959; Holland et al., 1994) avalia a confiança dos sujeitos para realizarem atividades assistenciais, de ensino, e de busca pelo bem-estar do próximo. Os achados corroboram os resultados de Nunes (2009), Noronha e Ottati (2010) e Sartori et al. (2010).

Em contrapartida, das demais correlações, os dois coeficientes menores são os mais inconsistentes teoricamente. O fator Convencional, por exemplo, é caracterizado pela crença de boa realização em atividades que exigem mínima habilidade nas relações interpessoais, o que não parece ser condizente com a dimensão da EAP, na qual o atendimento ao outro pode estar presente. De igual modo, o fator Realista é caracterizado pela autoeficácia em carreiras como mecânico de carros, inspetor, carpinteiro, eletricista, cozinheiro ou agricultor, ou ainda, pelo gosto de trabalhar mais com objetos do que com pessoas, o que também não parece relacionado às atividades de Ciências Biológicas.

Assim como ocorreu na dimensão Ciências Biológicas e da Saúde, em relação à dimensão Ciências Agrárias e Ambientais e o fator Social, todos os coefi- cientes foram significativos. Noronha et al. (2007) informam que a dimensão da EAP concentra atividades como analisar e controlar produtos industrializados, prevenir doenças em lavouras e rebanhos e, também, analisar e elaborar relatórios sobre impacto ambiental. Ao avaliar o fator Social (Nunes, 2009; Holland, 1959; Holland et al., 1994) verifica-se estar relacionado à confiança para realizar tarefas assistenciais, como a busca pelo bem-estar do próximo, as quais possibilitem usar habilidades de comunicação e de relacionamento interpessoal, destacando-se os valores humanistas e religiosos. Os achados informam que foi entre Ciências Agrárias (EAAOc) e o fator Social (EAP) que se encontrou a correlação de maior magnitude, e confirmam os resultados obtidos por Sartori et al. (2010), Noronha e Ottati (2010), e Nunes (2009). Dispõe-se com o fator Investigativo o segundo maior coeficiente, embora baixo, revelando a natureza intelectual. Pessoas com alta autoeficácia no fator tendem a exercer carreiras como: biólogo, geólogo, químico, assistente de laboratório, inspetor de produtos, técnico em medicina, dentre outras. Com os fatores Artístico e Realista foram obtidas as duas menores correlações, o que pode ser explicado teoricamente pela incompatibilidade das características entre eles e a dimensão $\mathrm{Ci}$ ências Agrárias e Ambientais, descrita anteriormente.

A dimensão Atividades Burocráticas da EAP se correlacionou com magnitude alta e positiva com o Convencional da EAAOc. De acordo com Noronha et al. (2007) a referida dimensão da EAP concentra atividades tais como controlar produtos industrializados; elaborar plano diretor; criar programas de computadores e interpretar dados numéricos; conduzir negociações, divulgar e vender softwares; coordenar operações fiscais e financeiras. No que se refere ao tipo Convencional (Nunes, 2009; Holland, 1959; Holland et al., 1994), convém destacar que ele apresenta características que permitem identificar a relação entre os construtos, uma vez que demonstram acreditar que podem se envolver com atividades burocráticas, tarefas repetitivas e financeiras, porém, evitam situações contraditórias ou que necessitem habilidades sociais. Tendem buscar poder, reconhecimento e status, a serem conformistas e autocontrolados. Os achados desta pesquisa corroboram os resultados obtidos por Sartori et al. (2010) em seus estudos, já que foi identificada correlação moderada entre a dimensão Atividades Burocráticas da EAP e o tipo Convencional do SDS. O mesmo ocorreu nos estudos de Nunes (2009), 
contudo a magnitude da correlação foi alta $(r=0,70$; $p<0,05)$.

Outro resultado importante foi a correlação moderada entre Atividades Burocráticas e o fator Empreendedor. A dimensão Atividades Burocráticas concentra atividades como coordenar operações fiscais e financeiras, conduzir negociações e processos entre patrão e empregado. O tipo Empreendedor (Nunes, 2009; Holland, 1959; Holland et al., 1994) reúne pessoas com confiança nas habilidades para argumentar, apresenta bom contato social (o que não é tão marcante naqueles que preferem atividades burocráticas), evita tarefas repetitivas, capacidade para realizar vendas e atuar como líder. Os resultados são concordantes com Nunes (2009), Sartori et al. (2010) e Noronha e Ottati (2010).

Em relação à dimensão Ciências Humanas e Sociais Aplicadas (EAP) foi encontrada correlação moderada e positiva com o fator Social da EAAOc, o que é pertinente sob a perspectiva teórica. De acordo com Noronha et al. (2007) esta dimensão da EAP avalia interesses por atividades como conduzir relações entre empresa e empregados, estudar origem e evolução do homem e da cultura, colaborar na elaboração de programas educacionais. Em outra medida, o tipo Social (Nunes, 2009; Holland, 1959; Holland et al., 1994), retrata que os sujeitos exibem maior confiança para realizar atividades assistenciais, de ensino, de busca pelo bem-estar do próximo. Em comum, dimensão e fator possuem habilidades comunicativa e interpessoal, destacando-se valores humanistas e religiosos. Todavia, estão previstos afastamento de atividades que demandem o uso de força física. Os resultados identificados neste estudo estão alinhados com a pesquisa de Sartori et al. (2010) e Nunes (2009), que também observaram magnitudes semelhantes.

Outros coeficientes menores foram encontrados entre a dimensão e os fatores Artístico, Empreendedor e Convencional da EAAOc, possivelmente associados pela natureza interpessoal deles, presente em maior ou menor grau. Resultado não esperado, no entanto, se traduziu na baixa correlação entre Ciências Humanas e o fator Investigativo. Aventa-se como hipótese uma particularidade da amostra estudada.

Por fim, a dimensão Entretenimento apresentou duas correlações de moderada magnitude com os fatores Social e Entretenimento. A dimensão Entretenimento avalia interesses por produzir desfiles, editoriais de moda e campanhas publicitárias; atender hóspedes e turistas; gerenciar hotéis e parques temáticos (Noronha e cols., 2007). Os sujeitos que endossam o fator Social da EAAOc (Nunes, 2009; Holland, 1959; Holland et al., 1994) são aqueles que demonstram confiança em realizar tarefas assistenciais, de busca pelo bem-estar do próximo, bem como ao fazer uso de habilidades de comunicação e de relacionamento interpessoal; enquanto o fator Artístico demonstra o quanto sentem-se confiantes para usar a sensibilidade e criatividade em detrimento das tarefas concretas e burocráticas, por meio da autoexpressão em atividades artísticas.

Entretenimento também se correlacionou com os fatores de autoeficácia Empreendedor e Convencional. A relação com Empreendedor foi maior, embora baixa, e mais justificável sob a perspectiva teórica, uma vez que é possível inferir que tal autoeficácia envolva preferência por atividades associadas ao Entretenimento, como os relativos à elaboração de eventos, por exemplo.

As correlações entre as dimensões da EAP e as fontes de autoeficácia referentes à segunda seção da EAAOc estão dispostas na Tabela 2. As fontes são analisadas para identificar os fatores que contribuíram para o desenvolvimento das crenças de autoeficácia avaliadas na primeira seção do instrumento. 
Tabela 2

Correlação de Pearson ( $r$ ) entre as Dimensões da EAP e as Fontes de Autoeficácia da EAAOc ( $N=172)$

\begin{tabular}{ccccc} 
& \multicolumn{4}{c}{ Fontes de Autoeficácia da EAAOc } \\
& Experiências Autênticas & Aprendizagem Vicária \\
\hline Dimensões da EAP & $\boldsymbol{r}$ & $\boldsymbol{p}$ & $\boldsymbol{r}$ & $\boldsymbol{p}$ \\
\hline Ciências Exatas & 0,17 & $0,031^{*}$ & 0,10 & 0,187 \\
Artes e Comunicação & 0,17 & $0,030^{*}$ & 0,05 & 0,524 \\
Ciências Biológicas e da Saúde & 0,19 & $0,011^{*}$ & 0,27 & $0,000^{* *}$ \\
Ciências Agrárias e Ambientais & 0,18 & $0,021^{*}$ & 0,17 & $0,025^{*}$ \\
Atividades Burocráticas & 0,20 & $0,010^{*}$ & 0,18 & $0,018^{*}$ \\
Ciências Humanas e Sociais Aplicadas & 0,08 & 0,320 & 0,13 & 0,103 \\
Entretenimento & 0,08 & 0,273 & 0,18 & $0,017^{*}$ \\
\hline
\end{tabular}

$* p<0,05 ; * * p<0,001$

A Tabela 2 permitiu identificar a ocorrência de 9 correlações estatisticamente significativas de 14 possíveis, porém, 8 com magnitude muito baixa variando entre 0,17 e 0,27 . A maior magnitude se deu entre a dimensão Ciências Biológicas e da Saúde da EAP e Aprendizagem Vicária da EAAOc.

Ao analisar a referida correlação que obteve destaque, pode-se verificar de acordo com Noronha et al. (2007) que a dimensão da EAP avalia interesses, tais como realizar cirurgias e investigar a natureza e a causa de doenças. O fator da EAAOc (Bandura, 1977; 1986; 1997; Nunes, 2009) concentra aspectos geradores das crenças, os quais estão relacionados à observação de sujeitos realizando atividades que se tornam padrões de referência, passando a levar os observadores a acreditarem que também podem ter sucesso nas mesmas atividades. Portanto, tal fato pode ter ocorrido devido a probabilidade dessa fonte ter exercido influência na formação das crenças, pois os indivíduos possivelmente possuem dúvidas sobre sua capacidade, o que pode ocorrer devido à falta de experiência pessoal na atividade.

Os achados permitem inferir que há relação entre fontes de autoeficácia e interesses profissionais, embora os coeficientes encontrados tenham sido de muito baixa e baixa magnitude. $\mathrm{O}$ ocorrido pode ser interpretado com base nos conceitos apresentados por Bandura (1997), no que diz respeito ao desenvolvimento das crenças de autoeficácia, ou seja, a criação ocorre a partir das fontes, as quais não atuam diretamente sobre os interesses. No mesmo sentido, Lent et al. (1994) afirmam que as fontes antecedem a formação das crenças de autoeficácia, todavia não agem diretamente na formação dos interesses. Dessa forma, são as crenças que estão diretamente relacionadas ao desenvolvimento dos interesses profissionais.

\section{CONSIDERAÇÕES FINAIS}

O presente estudo destinou-se a investigar as relações entre dois testes que avaliam os construtos interesses profissionais e autoeficácia, sendo que no último foram consideradas as atividades e as fontes, em um grupo de tecnólogos, com vistas à busca de evidências de validade, considerando uma amostra ainda não pesquisada. Foram identificadas correlações entre os interesses profissionais e a autoeficácia para atividades ocupacionais, bem como, em relação às suas fontes. Como esperado, as relações entre os interesses e as atividades (primeira seção do instrumento de autoeficácia) geraram coeficientes de magnitudes maiores, do que a segunda seção (fontes de autoeficácia).

Acredita-se que o propósito desta pesquisa foi alcançado em virtude das correlações obtidas e das discussões que puderam ser promovidas. Essas constatações podem ser fundamentadas com base nas asserções de Lent et al. (1994) e Bandura (2001), que afirmam que a autoeficácia se relaciona com a formação dos interesses profissionais. Nesse ensejo, os resultados do presente estudo reafirmam a relação entre os construtos avaliados pelas duas escalas.

Trabalhos dessa natureza ainda são necessários no Brasil, pois em que pese o fato da orientação profissi- 
onal estar revendo seus paradigmas em razão das demandas do mercado de trabalho (Sparta, Bardagi, \& Teixeira, 2006), os instrumentos utilizados devem se atualizar e acompanhar as novas necessidades. Há que se ressaltar a asserção de Melo-Silva, Leal e Fracalozzi (2010), no sentido de que os processos de orientação profissional podem minimizar as dificuldades relativas à escolha profissional. Sob esta perspectiva, tal como pontuado por Noronha, Sisto e Santos (2007) a escolha refletida tende a trazer benefícios pessoais e sociais.

Adicionalmente, as pesquisas desenvolvidas com os instrumentos em questão, não incluíram em suas amostras, os estudantes de tecnologia (Sartori et al., 2010; Nunes, 2009; Nunes \& Noronha, 2011). No entanto, há que se ressaltar que a amostra desta pesquisa foi escolhida por conveniência e apresenta limitações, uma vez que contempla estudantes de apenas três cursos de uma mesma instituição. Nesse sentido, sugere-se realizar estudos futuros em diferentes instituições de ensino superior que formem tecnólogos em outras áreas de atuação profissional, para aprofundar a compreensão sobre o tema em questão, assim como se recomenda a utilização de instrumentos e construtos diversificados.

\section{REFERÊNCIAS}

Ambiel, R. A. M. (2010). Construção da Escala de AutoEficácia para Escolha Profissional. Dissertação de Mestrado, USF, Itatiba.

American Educational Research Association (AERA), American Psychological Association (APA), National Council on Measurement in Education (NCME) (1999). Standards for Educational and Psychological Testing. Washington, DC: American Educational Research Association.

Bandura, A. (1977). Self-efficacy: Toward a unifying theory of behavior change. Psychological Review, 84, 191215.

Bandura, A. (1986). Social foundations of thought and action: A social cognitive theory. Englewood Cliffs, NJ: Prentice-Hall.

Bandura, A. (1997). Self-efficacy: the exercise of control. New York: W. H. Freeman and Company.

Bandura, A. (2001). Social Cognitive Theory: an agentic perspective. Annual Review of Psychology, 52(1), 1-26.

Betz, N. E., \& Borgen, F. H. (2000). The Future of Career Assessment: Integrating Vocational Interests with Self-
Efficacy and Personal Styles. Journal of Career Assessment, 8(4), 329-338.

Brasil (2010a). Ministério da Educação/Secretaria de Educação Profissional e Tecnológica. Catálogo Nacional de Cursos Superiores de Tecnologia. Brasília.

Brasil (2010b). Ministério da Educação. Educação Profissional. Diploma dos tecnólogos vale para concurso e pósgraduação, de 22 de julho de 2010 . Retirado em 21 de agosto de 2014, de http://portal.mec.gov.br/index.php? op tion=com_content\&view=article\&id=15698:diplomados-tecnologos-vale-para-concurso-e-posgraduacao\& catid $=209$.

Bueno, J. M. H., Lemos, C. G., \& Tomé, F. A. M. F. (2004). Interesses profissionais de um grupo de estudantes de psicologia e suas relações com inteligência e personalidade. Psicologia em Estudo, 9(2), 271-278.

Conselho Federal de Psicologia - CFP. Resolução 02/03. Retirado em 15 de novembro de 2010 de http://www.pol.org.br.

Conselho Federal de Psicologia - CFP. Resolução 25/01. Retirado em 15 de novembro de 2010 de http://www.pol.org.br.

Holland, J. L. (1959). A theory of vocational choice. Journal of Counseling Psychology, 6, 35-45.

Holland, J. L. (1977). Vocational indecision: more evidence and speculation. Journal of Counseling Psychology, 24, 404-414.

Holland, J. L., Fritzsche, B. A., \& Powell, A. B. (1994). SDS - Self-Directed Search. Los Angeles, California: PAR - Psychological Assessment Resources.

Lent, R., Brown, S. D., \& Hackett, G. (1994). Toward a unifying social cognitive theory of career and academic interest, choice and performance. Journal of Vocational Behavior, 45, 79-122.

Levenfus, R. S., \& Soares, D. H. P. (2002). Orientação Vocacional Ocupacional: Novos achados teóricos, técnicos e instrumentais para a clínica, a escola e a empresa. Porto Alegre: Artmed.

Melo-Silva, L. L., Leal, M. S., \& Fracalozzi, N. M. N. (2010). Produção científica em congressos brasileiros de orientação vocacional e profissional: Período 1999-2009. Revista Brasileira de Orientação Profissional, 11(1), 107-120.

Noronha, A. P. P., \& Ambiel, R. A. M. (2008). Fontes de Eficácia e Interesses Profissionais: relações entre pais e filhos. Evaluar, 8, 32-45.

Noronha, A. P. P., \& Ambiel, R. A. M. (2006). Orientação profissional e vocacional: análise da produção científica. Psico-USF, 11(1), 75-84. 
Noronha, A. P. P., Sisto, F., \& Santos, A. A. A. (2007). Escala de Aconselhamento Profissional - EAP - Manual Técnico. Itatiba-SP: Vetor Editora.

Noronha, A. P. P., \& Ottati, F. (2010). Interesses profissionais de jovens e escolaridade dos pais. Revista Brasileira de Orientação Profissional, 11(1), 37-47.

Noronha, A. P. P.; Santos, A. A. A.; Sisto, F. F. (2010). Contribuições da Escala de Aconselhamento Profissional (EAP) para a Orientação de Carreira. Em R. S. Levenfus, \& D. H. P. Soares (Orgs.). Orientação Vocacional Ocupacional: Novos Achados Teóricos, Técnicos e Instrumentais para a Clínica, a Escola e a Empresa (pp. 183193). Porto Alegre: Artmed.

Nunes, M. F. O. (2009). Estudos Psicométricos da Escala de Auto-Eficácia para Atividades Ocupacionais. Tese de Doutorado, USF, Itatiba.

Nunes, M. F. O., \& Noronha, A. P. P. (2009). Modelo Sócio-Cognitivo para a Escolha de Carreira: O papel da auto-eficácia e de outras variáveis relevantes. Educação Temática Digital, 10 (esp.), 16-35.

Nunes, M. F. O., \& Noronha, A. P. P. (2011). Associações entre autoeficácia para atividades ocupacionais e interesses em adolescentes. Psicologia: Reflexão e Crítica, 24(1), 1-9.

Pajares, F. (2002). Overview of Social Cognitive Theory and Self-efficacy. Retirado em 07 de junho de 2012 de http://www.emory.edu/education/mpf/eff.html.

Sartori, F. A., Noronha, A. P. P., Godoy, S., \& Ambiel, R. A. M. (2010). Interesses Profissionais de Jovens de Ensino Médio: estudo correlacional entre a escola de acom- panhamento profissional e o self-directed search carrier explorer. Estudos de Psicologia, 27(2), 215-225.

Savickas, M. L. (1995). Examining the Personal Meaning of Inventoried Interests During Counseling. Journal of Career Assessment, 3(2), 188-201.

Sisto, F. F. (2007). Delineamento Correlacional. Em M. N. Baptista \& D. C. Campos (Orgs.). Metodologias de Pesquisa em Ciências: análises quantitativa e qualitativa (pp. 90-101). Rio de Janeiro: LTC.

Sparta, M., Bardagi, M. P., \& Teixeira, M. A. P. (2006). Modelos e Instrumentos de Avaliação em orientação profissional: perspectiva histórica e situação no Brasil. Revista Brasileira de Orientação Profissional, 7(2), 1932.

Teixeira, M. A. P., Lassance, M. C. P., Silva, B. M. B., \& Bardagi, M. P. (2007). Produção científica em orientação profissional: uma análise da revista brasileira de orientação profissional. Revista Brasileira de Orientação Profissional 8(2), 25-40. 
